\section{TATRA \\ MOUNTaiNS \\ Mathematical Publications}

DOI: $10.2478 / \mathrm{tmmp}-2013-0014$

Tatra Mt. Math. Publ. 55 (2013), 1-16

\title{
GENERALIZED DISCONTINUITY OF REAL-VALUED FUNCTIONS
}

\author{
RAFAE ZDUŃCZYK
}

\begin{abstract}
We present a proof of the theorem on countability of the set of points of generalized discontinuity of an $(\mathcal{S}, \mathcal{Y})$-regular real function $f: X \rightarrow \mathbb{R}$, where $\mathcal{S}$ is a local system in $X$ and $\mathcal{Y}$ is a partition of $X$. We start with a definition of a local system in a generalized form and with basic properties of local systems. The concepts are illustrated with examples. The main result is applied both for regularities in the sense of density connected with the Lebesgue measure on $\mathbb{R}^{n}$ (Lebesgue density) and with Baire category $(\mathcal{I}$-density), respectively.
\end{abstract}

\section{Introduction}

Let $(X, \mathcal{T})$ be a topological space. Any $U \subset X$ such that $x \in \mathcal{T}$-int $U$ will be called a $\mathcal{T}$-neighbourhood of $x$.

Definition. Let $(X, \mathcal{T})$ be dense-in-itself (i.e., there are no $\mathcal{T}$-isolated points in $X)$. Any class $\mathcal{S}=\{S(x)\}_{x \in X}$, where each $S(x)$ is a collection of subsets of $X$, will be referred to as a local system in $(X, \mathcal{T})$ (or simply, a local system, when confusion is unlikely), if for any $x \in X$ the following conditions hold:

1. (a) $\{x\} \notin S(x)$,

(b) $S(x) \neq \varnothing$,

2. if $S \in S(x)$, then $x \in S$,

3. if $S_{1} \in S(x)$ and $S_{1} \subset S_{2}$, then $S_{2} \in S(x)$,

4. if $x \in U, U \in \mathcal{T}$ and $S \in S(x)$, then $S \cap U \in S(x)$.

Remark 1. If $x_{0} \in \operatorname{int} U$ and $\{S(x)\}_{x \in X}$ is a local system, then $U \in S\left(x_{0}\right)$, by 4 .

(C) 2013 Mathematical Institute, Slovak Academy of Sciences.

2010 Mathematics Subject Classification: 54C08, 54C30, 28A10, 26A15, 54E35.

Keywords: local system, filtering system, generalized limits, density point, $\mathcal{I}$-density point, lower density. 
The hereby defined local systems are a generalization up to topological spaces of a concept of Brian Thomson of 1980's (compare [10, p. 3, (2.1)] and [9. p. $280,(1.1)]$ ), originally formulated for the real line equipped with the Euclidean topology. In the paper [8], the same notion is considered but the term local system or any other is not attached to it there. Certainly, the generalization has its limitations, for if $X$ had isolated points, then conditions 1 and 4 would be contradictory. Therefore we make a general assumption on $(X, \mathcal{T})$ to be dense-in-itself.

EXAMPLE 1.

1. If $2 \leqslant \mathfrak{m} \leqslant \operatorname{card} X$, then the collections

$$
\mathcal{S}_{\mathfrak{m}}:=\{\{S \subset X: x \in S \text { and } \operatorname{card} S \geqslant \mathfrak{m}\}: x \in X\}
$$

are distinct local systems in $(X,\{\varnothing, X\})$.

2. In each dense-in-itself topological space class $\mathcal{S}_{0}:=\left\{S_{0}(x)\right\}_{x \in X}$, where $S \in S_{0}(x)$, if $S$ is a $\mathcal{T}$-neighbourhood of $x$, forms a local system. Moreover, if $\mathcal{T}=\{\varnothing, X\}$, then $S_{0}(x)=\{X\}$, for any $x \in X$.

3. From now on, let $A^{d}$ stand for the $\mathcal{T}$-derived set of $A$. Then

$$
\mathcal{S}_{\infty}:=\left\{S_{\infty}(x)\right\}_{x \in X},
$$

where $S \in S_{\infty}(x)$, if $x \in S^{d} \cap S$, is a local system as well. In particular, $\mathcal{S}_{\infty}$ in $(X,\{\varnothing, X\})$ coincides with $\mathcal{S}_{2}$ defined in 1 .

4. Let $\mathcal{Y}:=\left\{Y_{x}: x \in X\right\}$ be a collection of $Y_{x} \subset X$ such that $x \in\left(Y_{x}\right)^{d}$. Put $S_{\mathcal{Y}, 0}(x):=\left\{S \subset X: x \in S \supset U_{x} \cap Y_{x}\right.$, where $U_{x}$ is a $\mathcal{T}$-neighbourhood of $\left.x\right\}$ and

$$
S_{\mathcal{Y}, \infty}(x):=\left\{S \subset X: S_{x} \cup\{x\} \subset S \text {, for some } S_{x} \subset Y_{x} \text {, such that } x \in\left(S_{x}\right)^{d}\right\} \text {. }
$$

Then the collections

$$
\mathcal{S}_{\mathcal{Y}, 0}:=\left\{S_{\mathcal{Y}, 0}(x): x \in X\right\} \quad \text { and } \quad \mathcal{S}_{\mathcal{Y}, \infty}:=\left\{S_{\mathcal{Y}, \infty}(x): x \in X\right\}
$$

are local systems in $(X, \mathcal{T})$. Note that for $Y_{x} \equiv X$ we have $\mathcal{S}_{\mathcal{Y}, \infty}=\mathcal{S}_{\infty}$ and $\mathcal{S}_{\mathcal{Y}, 0}=\mathcal{S}_{0}$.

Any system such that all the $S(x)$ 's are stable under finite intersections will be called a filtering local system. The class of all filtering local systems in $(X, \mathcal{T})$ will be denoted by $\mathbb{F} \mathbb{S}(X, \mathcal{T})$.

ExAmple 2. $\mathcal{S}_{0} \in \mathbb{F} \mathbb{S}(X, \mathcal{T})$, for every $(X, \mathcal{T})$.

EXAMPLE 3 .

1. If card $X>2$, the system $\mathcal{S}_{2}$ from Example 111 is not filtering.

2. If card $X=2$, we have $\mathcal{S}_{0}=\mathcal{S}_{\infty}$, hence, in this case, $\mathcal{S}_{\infty} \in \mathbb{F} \mathbb{S}(X, \mathcal{T})$. 


\section{GENERALIZED DISCONTINUITY OF REAL-VALUED FUNCTIONS}

ExAmPLE 4. Let $\mathcal{T}_{\text {nat }}$ stand for the Euclidean topology. Then, $\mathcal{S}_{\infty} \notin \mathbb{F} \mathbb{S}\left(\mathbb{R}^{n}, \mathcal{T}_{\text {nat }}\right)$.

TheOREM 1. Assume that $X$ is infinite or finite with even cardinality. There is a topology $\mathcal{T}_{\text {ev }}$ on $X$ such that $\mathcal{S}_{\infty} \in \mathbb{F} \mathbb{S}\left(X, \mathcal{T}_{\text {ev }}\right)$.

P r o of. Let $\mathcal{T}_{\text {ev }}$ be a topology on $X$ introduced by the basis $\mathcal{U}$ consisting of disjoint two-element sets. Hence,

$$
S_{\infty}(x)=\left\{S: U_{x} \subset S\right\}, \quad x \in X,
$$

where $U_{x} \in \mathcal{U}$ is a basic $\mathcal{T}_{e v}$-neighbourhood of $x$. Note that in $\left(X, \mathcal{T}_{\text {ev }}\right)$ we have $\mathcal{S}_{0}=\mathcal{S}_{\infty}$. Thus, by Example $2, \mathcal{S}_{\infty} \in \mathbb{F} \mathbb{S}\left(X, \mathcal{T}_{\text {ev }}\right)$.

For local systems $\mathcal{S}^{\prime}$ and $\mathcal{S}^{\prime \prime}$ in $(X, \mathcal{T})$, let us define

$$
\mathcal{S}^{\prime} \sqcup \mathcal{S}^{\prime \prime} \quad \text { as } \quad\left\{S^{\prime}(x) \cup S^{\prime \prime}(x): x \in X\right\}
$$

and

$$
\mathcal{S}^{\prime} \sqcap \mathcal{S}^{\prime \prime} \quad \text { as } \quad\left\{S^{\prime}(x) \cap S^{\prime \prime}(x): x \in X\right\} .
$$

For a collection $\mathbb{S}$ of local systems in $(X, \mathcal{T})$, let $\bigsqcup \mathbb{S}, \Pi \mathbb{S}$ denote the collections

$$
\{\{S \in S(x), \quad \text { for some } \mathcal{S} \in \mathbb{S}\}: x \in X\}
$$

and

$$
\{\{S \in S(x), \quad \text { for all } \mathcal{S} \in \mathbb{S}\}: x \in X\},
$$

respectively.

Property 1. Assume that $\mathcal{S}^{\prime}$ and $\mathcal{S}^{\prime \prime}$ are local systems in $(X, \mathcal{T})$, and that $\mathbb{S}$ is a collection of local systems in $(X, \mathcal{T})$. Collections $\mathcal{S}^{\prime} \sqcup \mathcal{S}^{\prime \prime}, \mathcal{S}^{\prime} \sqcap \mathcal{S}^{\prime \prime}, \sqcup \mathbb{S}$ and $\Pi \mathbb{S}$ are well defined local systems.

Property 2. Given a local system $\mathcal{S}$ in $(X, \mathcal{T})$, we have:

$$
\mathcal{S} \sqcap \mathcal{S}=\mathcal{S}=\mathcal{S} \sqcup \mathcal{S} \text {. }
$$

Corollary 1. Let $\mathbb{S}(X, \mathcal{T})$ denote the collection of all local systems of a fixed dense-in-itself $(X, \mathcal{T})$. The structure $\mathfrak{S}:=\left(\mathbb{S}(X, \mathcal{T}), \sqcap, \sqcup, \mathcal{S}_{0}, \mathcal{S}_{\infty}\right)$ forms a lattice with $1=\mathcal{S}_{\infty}$ and $\mathrm{o}=\mathcal{S}_{0}$ (cf. [5]).

Property 3. $\Pi \mathbb{S} \in \mathbb{F} \mathbb{S}(X, \mathcal{T})$ provided $\mathbb{S} \subset \mathbb{F} S(X, \mathcal{T})$.

Remark 2. It may happen that all the systems from $\mathbb{S}$ are filtering, while $\bigsqcup \mathbb{S}$ is not. An appropriate example will be given in the sequel (Remark 5).

If $\mathcal{S}^{\prime}, \mathcal{S}^{\prime \prime}$ are local systems and $S^{\prime}(x) \subset S^{\prime \prime}(x)$ holds for each $x \in X$, then we write $\mathcal{S}^{\prime} \sqsubset \mathcal{S}^{\prime \prime}$.

Property 4. The relation " $\sqsubset$ " is a partial order in $\mathbb{S}(X, \mathcal{T})$. Moreover, for any $\mathbb{S} \subset \mathbb{S}(X, \mathcal{T})$ and $\mathcal{S} \in \mathbb{S}$, we have $\mathcal{S}_{0} \sqsubset \mathcal{S} \sqsubset \mathcal{S}_{\infty}, \sqcap \mathbb{S} \sqsubset \mathcal{S} \sqsubset \bigsqcup \mathbb{S}$.

The following is a straightforward consequence of this. 
Corollary 2. $\prod \mathbb{S}(X, \mathcal{T})=\mathcal{S}_{0}$ and $\bigsqcup \mathbb{S}(X, \mathcal{T})=\mathcal{S}_{\infty}$.

Property 5. If $\mathcal{S}^{\prime} \sqsubset \mathcal{S}^{\prime \prime}$, then $\mathcal{S}^{\prime} \sqcap \mathcal{S}^{\prime \prime}=\mathcal{S}^{\prime}, \mathcal{S}^{\prime} \sqcup \mathcal{S}^{\prime \prime}=\mathcal{S}^{\prime \prime}$.

Property 6. It is possible that $\mathcal{S}^{\prime \prime} \in \mathbb{F} \mathbb{S}(X, \mathcal{T}), \mathcal{S}^{\prime} \sqsubset \mathcal{S}^{\prime \prime}$, but $\mathcal{S}^{\prime} \notin \mathbb{F} \mathbb{S}(X, \mathcal{T})$. An appropriate example will be given in the sequel (Remark 5).

In the light of Property 6, it is clear that, for Property 3, the assumption that all the systems in $\mathbb{S}$ are filtering, is relevant and cannot be weakened.

Let $T$ be any set of indices. Let us assume that some sets $Y_{x}^{t} \subset X$, for $t \in T$ are related to each $x \in X$. Put $\mathcal{Y}_{x}:=\left\{Y_{x}^{t}: t \in T\right\}$ for $x \in X$ and $\mathcal{Y}:=\left\{Y_{x}^{t}: t \in T, x \in X\right\}$. If for each $x \in X$ the following conditions hold,

1. sets $Y_{x}^{t_{1}}, Y_{x}^{t_{2}},\{x\}$ are pairwise disjoint for all $t_{1}, t_{2} \in T, t_{1} \neq t_{2}$,

2. $X=\{x\} \cup \cup \mathcal{Y}_{x}$,

3. $x \in \bigcap_{t \in T}\left(Y_{x}^{t}\right)^{d}$,

then we call $\mathcal{Y}$ a partition of $(X, \mathcal{T})$. We will say that $\mathcal{S}=\{S(x): x \in X\}$ is $\mathcal{Y}$-decomposable if $S \cap Y_{x}^{t} \neq \varnothing$ for any $t \in T, x \in X$ and $S \in S(x)$.

TheOREM 2. If $\mathcal{S}$ is $\mathcal{Y}$-decomposable, then the collections $\mathcal{S}_{\mathcal{Y}}^{t}:=\left\{S_{\mathcal{Y}}^{t}(x): x \in X\right\}$, where

$$
S_{\mathcal{Y}}^{t}(x):=\left\{S \subset X: S \supset \hat{S} \cap\left(Y_{x}^{t} \cup\{x\}\right), \text { for some } \hat{S} \in S(x)\right\},
$$

are well defined local systems, for all $t \in T$.

P r o of. Let us fix $x \in X$ and $t \in T$. We omit proofs of $1-3$ and focus on point 4 being the only nontrivial one. Let us consider $S \cap U$, where $S \in S^{t}(x)$ and $x \in U \in \mathcal{T}$. The proof will be accomplished if we find $\hat{S}_{U} \in S(x)$, for which

$$
\hat{S}_{U} \cap\left(Y_{x}^{t} \cup\{x\}\right) \subset S \cap U .
$$

There is an $\hat{S} \in S(x)$ such that $\hat{S} \cap\left(Y_{x}^{t} \cup\{x\}\right) \subset S$. Put $\hat{S}_{U}:=\hat{S} \cap U$. It follows that $\hat{S}_{U} \in S(x)$ and that

$$
\hat{S}_{U} \cap\left(Y_{x}^{t} \cup\{x\}\right)=\hat{S} \cap\left(Y_{x}^{t} \cup\{x\}\right) \cap U \subset S \cap U .
$$

The proof is now complete.

Property 7. If $\mathcal{S}^{\prime}$ is $\mathcal{Y}$-decomposable and $\mathcal{S}^{\prime \prime} \sqsubset \mathcal{S}^{\prime}$, then $\mathcal{S}^{\prime \prime}$ is $\mathcal{Y}$-decomposable as well. $\mathcal{S}_{0}$ is $\mathcal{Y}$-decomposable, for all the partitions $\mathcal{Y}$.

In $\left(\mathbb{R}, \mathcal{T}_{\text {nat }}\right)$, let us consider the partition $\mathcal{Y}_{\infty}:=\left\{Y_{x}^{\prime}, Y_{x}^{\prime \prime}: x \in \mathbb{R}\right\}$, where $Y_{x}^{\prime}:=(-\infty ; x)$ and $Y_{x}^{\prime \prime}:=(x ; \infty)$. The $\mathcal{Y}_{\infty}$-decomposable systems will be shortly called bilateral. In $\left(\mathbb{R}, \mathcal{T}_{\text {nat }}\right)$, for $\mathcal{Y}_{\infty}$ and a bilateral $\mathcal{S}$, we introduce the following denotations:

$$
\begin{aligned}
& \mathcal{S}^{-}:=\left\{S^{-}(x): x \in \mathbb{R}\right\}, \quad \text { where } S^{-}(x):=\{(-\infty, x] \cap S: S \in S(x)\} ; \\
& \mathcal{S}^{+}:=\left\{S^{+}(x): x \in \mathbb{R}\right\}, \quad \text { where } S^{+}(x):=\{[x,+\infty) \cap S: S \in S(x)\} .
\end{aligned}
$$




\section{GENERALIZED DISCONTINUITY OF REAL-VALUED FUNCTIONS}

Example 5. Let us define a partition of $\left(\mathbb{R}, \mathcal{T}_{\text {nat }}\right)$ by the following. Put

$$
\begin{gathered}
A_{n}:=\left(-2^{-n} ;-2^{-n-1}\right] \cup\left[2^{-n-1} ; 2^{-n}\right), \quad n \in \omega, \\
Y_{x}^{2 \prime}:=x+\bigcup_{n \in \omega} A_{2 n}, \quad Y_{x}^{2 \prime \prime}:=x+\left((-\infty ;-1] \cup[1 ; \infty) \cup \bigcup_{n \in \omega} A_{2 n+1}\right)
\end{gathered}
$$

and $\mathcal{Y}_{2}:=\left\{\left\{Y_{x}^{2 \prime}, Y_{x}^{2 \prime \prime}\right\}: x \in \mathbb{R}\right\}$. Let us consider two local systems:

$$
\mathcal{S}_{2 \omega}:=\left\{\left\{S: S \supset\{x\} \cup\left(x+\bigcup_{n>N} A_{2 n}\right), \text { for some } N \in \omega\right\}, x \in \mathbb{R}\right\}
$$

and

$$
\mathcal{S}_{+0}:=\left\{\left\{S: U \cap[x ; \infty) \subset S, x \in U \in \mathcal{T}_{\text {nat }}\right\}: x \in \mathbb{R}\right\} .
$$

It is easy to check that $\mathcal{S}_{+0}$ is $\mathcal{Y}_{2}$-decomposable but not $\mathcal{Y}_{\infty}$-decomposable, whilst $\mathcal{S}_{2 \omega}$, on the contrary, is $\mathcal{Y}_{\infty}$-decomposable but not $\mathcal{Y}_{2}$-decomposable.

THEOREM 3. For every $\mathcal{Y}$-decomposable local system $\mathcal{S}$,

$$
\mathcal{S} \sqsubset \prod_{t \in T} \mathcal{S}_{\mathcal{Y}}^{t}
$$

Moreover, if $\mathcal{S} \in \mathbb{F} \mathbb{S}(X, \mathcal{T})$ and $T$ is finite, then

$$
\mathcal{S}=\prod_{t \in T} \mathcal{S}_{\mathcal{Y}}^{t}
$$

Proof. The first part is trivial. For the proof of the latter, let us assume that $\operatorname{card} T=2, T=\{\prime, \prime \prime\}$ and that $\mathcal{S} \in \mathbb{F} \mathbb{S}(X, \mathcal{T})$. Let us fix $x \in X$ and $S$ from $S^{\prime}(x) \cap S^{\prime \prime}(x)$. Then

$$
\begin{aligned}
& S \supset S^{\prime} \cap\left(Y_{x}^{\prime \prime} \cup\{x\}\right), \\
& S \supset S^{\prime \prime} \cap\left(Y_{x}^{\prime} \cup\{x\}\right),
\end{aligned}
$$

for some $S^{\prime}, S^{\prime \prime} \in S(x)$. We are searching for $S_{0} \subset S$ such that $S_{0} \in S(x)$. To this end, put $S_{0}:=S^{\prime} \cap S^{\prime \prime}$. Certainly,

$$
S_{0}=\left(\left(S^{\prime} \cap S^{\prime \prime}\right) \cap\left(Y_{x}^{\prime} \cup\{x\}\right)\right) \cup\left(\left(S^{\prime} \cap S^{\prime \prime}\right) \cap\left(Y_{x}^{\prime \prime} \cup\{x\}\right)\right) \subset S .
$$

The proofs for higher but still finite cardinalities of $T$ are similar.

$\mathcal{Y}$-decomposable systems, for which condition (2) is fulfilled, will be referred to as $\mathcal{Y}$-retraceable.

The $\mathcal{Y}$-decomposable filtering system may fail to be $\mathcal{Y}$-retraceable when $T$ is infinite. 
ExAmPle 6 . Let us consider the system $\mathcal{S}_{0}$ in $\left(\mathbb{R}^{2}, \mathcal{T}_{\text {nat }}\right)$. We will define a partition

$$
\mathcal{Y}=\left\{Y_{x}^{j}: j \in \omega, x \in \mathbb{R}^{2}\right\},
$$

for which $\prod_{j \in \omega}\left(\mathcal{S}_{0}\right)_{\mathcal{Y}}^{j} \not \subset \mathcal{S}_{0}$. Let

and

$$
\varphi_{j}:=2 \pi\left(1-2^{-j}\right), \quad j \in \omega
$$

$$
z_{j}:=\left(\cos \varphi_{j}, \sin \varphi_{j}\right) \in \mathbb{R}^{2}, \quad j \in \omega .
$$

For every $x \in \mathbb{R}^{2}$, the collection of open rays $\left\{x+\left\{t z_{j}, t>0\right\}\right\}_{j \in \omega}$ induces a partition of $\left(\mathbb{R}^{2}, \mathcal{T}_{\text {nat }}\right)$ into denumerably many angular regions

$$
Y_{x}^{j}:=x+\left\{(t \cos \varphi, t \sin \varphi): t>0, \varphi \in\left[\varphi_{j} ; \varphi_{j+1}\right)\right\}, \quad j \in \omega .
$$

Let us fix an $x \in \mathbb{R}^{2}$ and consider $S:=\bigcup_{j \in \omega}\left(Y_{x}^{j} \cap B\left(x, 2^{-j}\right)\right.$ ), where (and hereafter) $B\left(x, 2^{-j}\right)$ is the open Euclidean ball centred at $x$ with radius $2^{-j}$. Clearly, $S \in\left(S_{0}\right)_{\mathcal{Y}}^{j}(x)$ for $j \in \omega$, but $x$ is not in int $S$, hence $S \notin S_{0}(x)$.

ExAmple 7. For a partition $\mathcal{Y}=\left\{Y_{x}^{t}: t \in T, x \in X\right\}$, where card $T \geqslant 4$, let $t_{1}, t_{2}, t_{3}, t_{4} \in T$ be fixed. Put $\bar{T}:=T \backslash\left\{t_{3}, t_{4}\right\}, \hat{T}:=T \backslash\left\{t_{1}, t_{2}, t_{3}, t_{4}\right\}$ and let the partition $\overline{\mathcal{Y}}:=\left\{Y_{x}^{t}: t \in \bar{T}, x \in X\right\}$ be defined by the following

$$
\bar{Y}_{x}^{t}:=\left\{\begin{array}{ll}
Y_{x}^{t}, & t \in \hat{T}, \\
Y_{x}^{t_{1}} \cup Y_{x}^{t_{2}}, & t=t_{1}, \\
Y_{x}^{t_{3}} \cup Y_{x}^{t_{4}}, & t=t_{2},
\end{array} \quad x \in X .\right.
$$

We will find a $\overline{\mathcal{Y}}$-decomposable system not fulfilling (2), by which we shall prove that for Theorem 3 the filtering assumption is vital. Let us consider

$$
\overline{\mathcal{S}}:=\left(\left(\mathcal{S}_{\overline{\mathcal{Y}}^{t, 0}} \sqcap \mathcal{S}_{\overline{\mathcal{Y}}^{t}, \infty}\right) \sqcup\left(\mathcal{S}_{\overline{\mathcal{Y}}^{t^{2}, 0}} \sqcap \mathcal{S}_{\overline{\mathcal{Y}}^{t_{1}, \infty}}\right)\right) \sqcap \prod_{t \in \hat{T}} \mathcal{S}_{\overline{\mathcal{Y}}^{t}, \infty},
$$

and the set $S_{x}$ defined as:

$$
S_{x}:=X \backslash\left(Y_{x}^{t_{2}} \cup Y_{x}^{t_{4}}\right) .
$$

It is clear that $\overline{\mathcal{S}}$ is $\overline{\mathcal{Y}}$-decomposable (and $\mathcal{Y}$-decomposable as well) and that $S_{x} \in \bigcap_{t \in \bar{T}} \bar{S}_{\overline{\mathcal{Y}}}^{t}(x) \backslash \bar{S}(x)$, which is a consequence of the relations $\{x\} \cup Y_{x}^{t_{1}} \in$ $\mathcal{S}_{\overline{\mathcal{Y}}^{t_{1}, \infty}},\{x\} \cup Y_{x}^{t_{3}} \in \mathcal{S}_{\overline{\mathcal{Y}}^{t_{2}, \infty}}$ and the equality $\overline{\mathcal{S}}_{\overline{\mathcal{Y}}}^{t}=\mathcal{S}_{\overline{\mathcal{Y}}^{t}, \infty}$, valid for $t \in \bar{T}$.

EXAMPLE 8. Let us consider the system $\mathcal{S}^{\circ}$ in $\left(\mathbb{R}^{n}, \mathcal{T}_{\text {nat }}\right), n \in \omega$, such that $S \in S^{\circ}(x)$ if $x \in \mathbb{R}^{n}$ is an inner point of the intersection of $S$ with each line $P_{x}$ passing through $x$. Let $\left\{P_{x}^{t}\right\}^{t \in T}$ denote the collection of all lines going through $x$. Obviously, $\mathcal{Y}^{P}:=\left\{P_{x}^{t} \backslash\{x\}\right\}_{x \in \mathbb{R}^{n}}^{t \in T}$ is a partition of $\left(\mathbb{R}^{n}, \mathcal{T}_{\text {nat }}\right)$ and card $T=2^{\omega}$. Moreover, system $\mathcal{S}^{\circ}$ is $\mathcal{Y}^{P}$-retraceable. This proves that the finiteness of $T$ is not necessary for a local system to be retraceable. 


\section{GENERALIZED DISCONTINUITY OF REAL-VALUED FUNCTIONS}

ExAmple 9 . In $\left(\mathbb{R}^{n}, \mathcal{T}_{\text {nat }}\right)$, for $n \in \omega$, let us define the partition $\mathcal{Y}^{\square}:=\left\{Y_{x}^{m}: m \in\right.$ $\left.\left\{1, \ldots, 2^{n}\right\}, x \in \mathbb{R}^{n}\right\}$, an $n$-dimensional version of partition $\mathcal{Y}_{\infty}$. Let $Z\left(a_{1}, \ldots, a_{n}\right)$ denote the Cartesian product of $n$ rays,

$$
Z\left(a_{1}, \ldots, a_{n}\right):=\underset{k=1}{\times}(-1)^{a_{k}}(0 ; \infty), \quad \text { for } \quad a_{k} \in\{0,1\}, 1 \leqslant k \leqslant n .
$$

Note that the function $\bar{m}$ defined by $\bar{m}\left(a_{1}, \ldots, a_{n}\right):=\sum_{k=1}^{n} a_{k} 2^{k-1}$ states a one-to-one correspondence between $\{0,1\}^{n}$ and $\left\{1, \ldots, 2^{n}\right\}$. Let us put

$$
\begin{aligned}
& Y_{x}^{1}:=x+(0, \infty)^{n}, \\
& Y_{x}^{\bar{m}\left(a_{1}, \ldots, a_{n}\right)}:=x+\left(\operatorname{cl} Z\left(a_{1}, \ldots, a_{n}\right) \backslash\left(\{0\} \cup \underset{m<\bar{m}\left(a_{1}, \ldots, a_{n}\right)}{\bigcup} Y_{0}^{m}\right)\right),
\end{aligned}
$$

for $\bar{m}\left(a_{1}, \ldots, a_{n}\right)>1$. Hence, the sets $Y_{x}^{m}$ are defined for all $m \in\left\{1, \ldots, 2^{n}\right\}$. It is easy to see that for each $x \in \mathbb{R}^{n}, \mathbb{R}^{n}=\{x\} \cup \bigcup_{m=1}^{2^{n}} Y_{x}^{m}$ and that the collection $\mathcal{Y}^{\square}$ is a well defined partition of $\left(\mathbb{R}^{n}, \mathcal{T}_{\text {nat }}\right)$.

Property 8. If $\mathcal{S} \in \mathbb{F} S(X, \mathcal{T})$ is $\mathcal{Y}$-decomposable, then all the $\mathcal{S}_{\mathcal{Y}}^{t}$ 's, for $t \in T$, are filtering as well.

P r o of. Let $\mathcal{S} \in \mathbb{F} S(X, \mathcal{T})$ be $\mathcal{Y}$-decomposable. Let us fix $x \in X, t \in T$ and $S_{1}, S_{2} \in S_{\mathcal{Y}}^{t}(x)$. Then, by definition there exist $S_{j}^{t} \in S(x), j=1,2$, for which

$$
S_{j} \supset S_{j}^{t} \cap\left(Y_{x}^{t} \cup\{x\}\right), \quad j=1,2 .
$$

Hence, according to the assumption on $\mathcal{S}$, we have $S_{\mathcal{Y}}^{t}:=S_{1}^{t} \cap S_{2}^{t} \in S(x)$ and the inclusion

$$
S_{1} \cap S_{2} \supset S^{t} \cap\left(Y_{x}^{t} \cup\{x\}\right),
$$

is fulfilled. Thus $\mathcal{S}_{\mathcal{Y}}^{t}$ is filtering.

Partition $\mathcal{Y}=\left\{Y_{x}^{t}\right\}_{x \in X}^{t \in T}$ is said to be regular, if

$$
R_{\mathcal{Y}}(x, y):=\bigcap_{t^{\prime} \in T} \bigcup_{t \neq t^{\prime}}\left(Y_{x}^{t} \cup Y_{y}^{t}\right) \neq \varnothing,
$$

for all pairs of $x, y \in X$, such that $x \neq y$.

Example 10. The partition $\mathcal{Y}^{\square}$ from Example 9 is regular.

Let $\mathcal{C}:=\{C(x, y): x, y \in X\}$, where

$$
X \supset C(x, y) \backslash\{x, y\} \neq \varnothing, \quad \text { for } x \neq y .
$$

Any such $\mathcal{C}$ will be called a regularity guardian of $\mathcal{Y}$ if

$$
C(x, y) \backslash\{x, y\} \subset R_{\mathcal{Y}}(x, y),
$$


for all $x, y \in X, x \neq y$. It follows that any regular system has a guardian. Obviously, if $\mathcal{Y}$ is regular and $\mathcal{C}_{1}$ is a guardian of its regularity and if $\mathcal{C}_{2}:=$ $\left\{C_{2}(x, y): x, y \in X\right\}$ is a collection satisfying (3) and such that

$$
C_{2}(x, y) \subset C_{1}(x, y), \quad \text { for all } \quad x, y \in X,
$$

then $\mathcal{C}_{2}$ is either a regularity guardian for $\mathcal{Y}$.

Property 9. Let $\mathcal{C}:=\{C(x, y): x, y \in X\}$ fulfill (3). The following conditions are equivalent:

- $\mathcal{C}$ is a guardian of regularity of $\mathcal{Y}$,

- $C(x, y) \subset \bar{C}(x, y)$, for all $x, y \in X, x \neq y$, for some $\overline{\mathcal{C}}=\{\bar{C}(x, y)\}$, a guardian of regularity of $\mathcal{Y}$,

- $C(x, y) \cap Y_{x}^{t} \cap Y_{y}^{t}=\varnothing$, for all $x, y \in X, x \neq y$ and $t \in T$,

- $\mathcal{C}^{*}:=\{C(x, y) \backslash\{x, y\}, x, y \in X\}$ is a regularity quardian for $\mathcal{Y}$,

- $\mathcal{C}_{*}:=\{C(x, y) \cup\{x, y\}, x, y \in X\}$ is a regularity quardian for $\mathcal{Y}$.

The above property allows us to pay no more attention to whether $x$ and $y$ are elements of $C(x, y)$ or not.

EXAMPLE 11. In the Euclidean space $\mathbb{R}^{n}$, by a cube of size $k>0$, we understand an image of $\left\{x \in \mathbb{R}^{n}: 0 \leqslant x_{j} \leqslant 1, j=1, \ldots, n\right\}$ under $k$-scaled similarity. For arbitrary $x, y \in \mathbb{R}^{n}$ let $C_{\odot}(x, y)$ be the intersection of the cubes centred at $x$ and $y$, respectively, with sizes $2 \max _{1 \leqslant j \leqslant n}\left|y_{j}-x_{j}\right|$ and edges parallel to axes of coordinates.

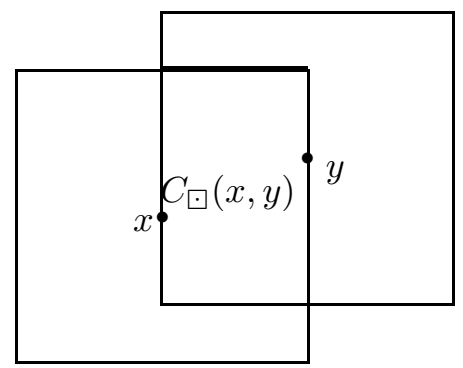

$\mathcal{C}_{\square}$ is a regularity guardian of $\mathcal{Y}^{\square}$. In particular, $C_{\square}(x, y)=[x ; y]$, for $n=1$.

ExAmple 12. Assume that $\varnothing \neq A \subsetneq \mathbb{R}$. Let us consider the partition $\mathcal{Y}_{\square}^{A}$ defined by letting

and

$$
Y_{x}^{A \prime}:=(-\infty ; x), \quad Y_{x}^{A \prime \prime}:=(x ;+\infty), \quad \text { if } x \in A,
$$

$$
Y_{x}^{A \prime \prime}:=(-\infty ; x), \quad Y_{x}^{A \prime}:=(x ;+\infty), \quad \text { if } x \notin A .
$$




\section{GENERALIZED DISCONTINUITY OF REAL-VALUED FUNCTIONS}

Partition $\mathcal{Y}_{\square}^{A}$ is regular, but the collection of intervals $\{[x ; y]: x, y \in \mathbb{R}\}$ is not its regularity guardian. Certainly, $\mathcal{C}_{\square}^{A}:=\left\{C_{\square}^{A}(x, y): x, y \in \mathbb{R}\right\}$, the collection of sets

$$
C_{\square}^{A}(x, y):= \begin{cases}(-\infty ; \min \{x, y\}] \cup[\max \{x, y\} ;+\infty), & \text { if } \operatorname{card}(\{x, y\} \cap A)=1, \\ {[x ; y],} & \text { else, }\end{cases}
$$

forms a regularity guardian of $\mathcal{Y}_{\square}^{A}$.

EXAMPLE 13.

1. In $\left(\mathbb{R}^{2}, \mathcal{T}_{\text {nat }}\right)$ let us define the partition $\overline{\mathcal{Y}}:=\left\{\bar{Y}_{x}^{\prime}, \bar{Y}_{x}^{\prime \prime}: x=\left(x_{1}, x_{2}\right) \in \mathbb{R}^{2}\right\}$ as

$$
\bar{Y}_{x}^{\prime}:=\left(\mathbb{R} \times\left(x_{2} ;+\infty\right)\right) \cup\left(\left(x_{1} ;+\infty\right) \times\left\{x_{2}\right\}\right)
$$

and

$$
\bar{Y}_{x}^{\prime \prime}:=\left(\mathbb{R} \times\left(-\infty ; x_{2}\right)\right) \cup\left(\left(-\infty ; x_{1}\right) \times\left\{x_{2}\right\}\right) .
$$

If $x$ and $y$ have different second coordinate $\left(x_{2} \neq y_{2}\right)$, then an open stripe $\mathbb{R} \times(\min \{x, y\} ; \max \{x, y\})$ is included in $R_{\bar{y}}(x, y)$. Moreover, for $x, y \in \mathbb{R}^{2}$ such that $x_{2}=y_{2}$, we have $R_{\bar{y}}(x, y)=\left(\min \left\{x_{1}, y_{1}\right\} ; \infty\right) \times\left\{x_{2}\right\}$, which means that $\overline{\mathcal{Y}}$ is regular. For all regularity guardians of $\overline{\mathcal{Y}}$, we have int $C(x, y)=\varnothing$, if $x_{2}=y_{2}$.

2. In $\left(\mathbb{R}^{2}, \mathcal{T}_{\text {nat }}\right)$ let us consider the partition $\tilde{\mathcal{Y}}:=\left\{\tilde{Y}_{x}^{\prime}, \tilde{Y}_{x}^{\prime \prime}: x \in \mathbb{R}^{2}\right\}$, where for $x=\left(x_{1}, x_{2}\right) \in \mathbb{R}^{2}, \tilde{Y}_{x}^{\prime}:=\left(\mathbb{R} \times\left[x_{2} ;+\infty\right)\right) \backslash\{x\}$ and $\tilde{Y}_{x}^{\prime \prime}:=\mathbb{R} \times\left(-\infty ; x_{2}\right)$. This partition fails to be regular.

The above examples might suggest that there is no partition of $\mathbb{R}^{2}$ into two parts with a guardian such that int $C(x, y) \neq \varnothing$. The following example refutes it.

ExAmple 14. For $x=\left(x_{1}, x_{2}\right) \in \mathbb{R}^{2}$ let us put

$$
\breve{Y}_{x}^{\prime}:=x+\left\{\left(\xi_{1}, \xi_{2}\right) \in \mathbb{R}^{2}: \xi_{2}>\xi_{1}^{2}\right\}
$$

and

$$
\breve{Y}_{x}^{\prime \prime}:=x+\left(\left\{\left(\xi_{1}, \xi_{2}\right) \in \mathbb{R}^{2}: \xi_{2} \leqslant \xi_{1}^{2}\right\} \backslash\{(0,0)\}\right) .
$$

Partition $\breve{\mathcal{Y}}$ is regular. If $\breve{C}(x, y):=\breve{Y}_{x}^{\prime} \triangle \breve{Y}_{y}^{\prime}$, then $\breve{\mathcal{C}}:=\left\{\breve{C}(x, y): x, y \in \mathbb{R}^{2}\right\}$ is a regularity guardian of $\breve{\mathcal{Y}}$ and all the $\breve{C}(x, y)$ 's have nonvoid interiors.

Assume that $(X, \rho)$ is a metric space, $\mathcal{C}=\{C(x, y): x, y \in X\}$ is a collection of subsets of $X$, which are nonvoid for $x \neq y$, and finally $\mathcal{S}=\{S(x): x \in X\}$, a local system in $(X, \mathcal{T})$, where $\mathcal{T}$ is topology introduced by $\rho$. If there exists $\delta:\left(z, S_{z}\right) \mapsto \delta_{S_{z}}^{z}>0$, with $S_{z} \in S(z)$, such that

$$
\underset{x, y \in X}{\forall} \underset{\substack{S_{x} \in S(x) \\ S_{y} \in S(y)}}{\forall}\left(\rho(y, x)<\min \left\{\delta_{S_{x}}^{x}, \delta_{S_{y}}^{y}\right\} \Rightarrow S_{x} \cap S_{y} \cap C(x, y) \neq \varnothing\right),
$$


then we say that the system $\mathcal{S}$ fulfills strong intersection condition with $\mathcal{C}-\operatorname{SIC}(\mathcal{C})$. If for every nonempty collection $\left\{S_{z}\right\}_{z \in X}$, such that $S_{z} \in S(z), z \in X$, there exists $\delta: z \mapsto \delta(z)>0$, for which

$$
\underset{x, y \in X}{\forall}\left(\rho(x, y)<\min \{\delta(x), \delta(y)\} \Rightarrow S_{x} \cap S_{y} \cap C(x, y) \neq \varnothing\right),
$$

then we say that $\mathcal{S}$ fulfills (ordinary) intersection condition with $\mathcal{C}-\mathrm{IC}(\mathcal{C})$. Obviously, $\operatorname{SIC}(\mathcal{C})$ implies $\mathrm{IC}(\mathcal{C})$. Examples of intersection conditions in $\left(\mathbb{R}, \mathcal{T}_{\text {nat }}\right)$ one may find in [2], 3] and in [9].

\section{Property 10.}

1. If $\mathcal{S}^{\prime \prime} \sqsubset \mathcal{S}^{\prime}$ and $\mathcal{S}^{\prime}$ fulfills (strong) intersection condition with $\mathcal{C}$, then $\mathcal{S}^{\prime \prime}$ also fulfills (strong) intersection condition with $\mathcal{C}$.

2. $\mathcal{S}_{0}$ fulfills strong intersection condition with $\mathcal{C}$ satisfying (3), providing $\{x, y\} \cap(C(x, y))^{d} \neq \varnothing$.

3. Let $\mathcal{C}:=\{C(x, y): x, y \in X\}$ satisfy (3). The following conditions are equivalent:

- system $\mathcal{S}$ fulfills (strong) intersection condition with $\mathcal{C}$,

- $C_{0}(x, y) \subset C(x, y)$, for all $x, y \in X$, for some $\mathcal{C}_{0}=\left\{C_{0}(x, y)\right\}$ satisfying (3) such that $\mathcal{S}$ fulfills (strong) intersection condition with $\mathcal{C}_{0}$.

P r o o f. To prove 1 , let $\delta^{\prime}$ be related to $\mathcal{S}^{\prime}$. It suffices to put $\delta^{\prime \prime}:=\delta_{\mid \bigcup\left(\{x\} \times S^{\prime \prime}(x)\right)}^{\prime}$.

To prove 2, put

$$
\delta\left(x, S_{x}\right):=\min \left\{\sup \left\{r>0: B(x, r) \subset S_{x}\right\}, 1\right\} \quad \text { for } \quad x \in X \text { and } S_{x} \in S_{0}(x) .
$$

Then, for some open $U$, we have

$$
\{x, y\} \subset U \subset B\left(x, \delta\left(x, S_{x}\right)\right) \cap B\left(y, \delta\left(y, S_{y}\right)\right) \subset S_{x} \cap S_{y},
$$

if $d(x, y)<\min \left\{\delta\left(x, S_{x}\right), \delta\left(y, S_{y}\right)\right\}$, and by assumption, $U \cap C(x, y) \neq \varnothing$.

Point 3 is obvious.

\section{Main result}

Now, we shall formulate a generalization of the results published in [4] and [14. Let us assume that $(X, \mathcal{T}),(Z, \tau)$ are topological spaces, $(X, \mathcal{T})$ - dense-in-itself. Let $\mathcal{S}$ be a local system in $(X, \mathcal{T}), f: X \rightarrow Z$ and $x \in X$. The set of all $c \in Z$ such that for $U_{c}$, an arbitrary neighbourhood of $c,\{x\} \cup f^{-1}\left(U_{c}\right) \in S(x)$, is denoted by $(\mathcal{S})-\operatorname{Lim}_{y \rightarrow x} f(y)$. In some cases $(\mathcal{S})-\operatorname{Lim}_{y \rightarrow x} f(y)$ may be a singleton, and then its only element is called $\mathcal{S}$-limit of $f$ at $x$, in notation $c=(\mathcal{S})-\lim _{y \rightarrow x} f(y)$. 


\section{GENERALIZED DISCONTINUITY OF REAL-VALUED FUNCTIONS}

Property 11. Assume that $(X, \mathcal{T})$ is dense-in-itself, $(Z, \tau)$ Hausdorff and $f: X \rightarrow Z$. The following conditions

1. $g=\lim _{y \rightarrow x} f(y)$,

2. $g=(\mathcal{S})-\lim _{y \rightarrow x} f(y)$,

3. $g \in\left(\mathcal{S}_{0}\right)-\operatorname{Lim}_{y \rightarrow x} f(y)$,

4. $g=\left(\mathcal{S}_{\infty}\right)-\lim _{y \rightarrow x} f(y)$

are related as follows:

1-3 are equivalent and imply 4 . If $(X, \mathcal{T})$ is first countable and $(Z, \tau)$ compact, then all 1-4 are equivalent.

Assuming $\mathcal{S}$ to be $\mathcal{Y}$-decomposable with $\mathcal{Y}=\left\{Y_{x}^{t}\right\}_{x \in X}^{t \in T}$, we introduce the following notation:

$$
\mathcal{S}_{\mathcal{Y}}^{\sqcap}:=\prod_{t \in T} \mathcal{S}_{\mathcal{Y}}^{t}, \quad \mathcal{S}_{\mathcal{Y}}^{\sqcup}:=\bigsqcup_{t \in T} \mathcal{S}_{\mathcal{Y}}^{t} .
$$

Let us note an obvious fact: $\mathcal{S}_{0} \sqsubset \mathcal{S} \sqsubset \mathcal{S}_{\mathcal{Y}} \sqsubset \mathcal{S}_{\mathcal{Y}} \sqsubset \mathcal{S}_{\infty}$. Hence we have

$$
\begin{aligned}
& \left(\mathcal{S}_{0}\right)-\operatorname{Lim}_{z \rightarrow x} f(z) \subset(\mathcal{S})-\operatorname{Lim}_{z \rightarrow x} f(z) \subset\left(\mathcal{S}_{\mathcal{Y}}^{\sqcap}\right)-\operatorname{Lim}_{z \rightarrow x} f(z) \\
& \subset\left(\mathcal{S}_{\mathcal{Y}}^{\sqcup}\right)-\operatorname{Lim}_{z \rightarrow x} f(z) \subset\left(\mathcal{S}_{\infty}\right)-\operatorname{Lim}_{z \rightarrow x} f(z) .
\end{aligned}
$$

Certainly, if $\mathcal{S}$ is $\mathcal{Y}$-retraceable, we have $\left(\mathcal{S}_{\mathcal{Y}}\right)-\operatorname{Lim}_{z \rightarrow x} f(z)=(\mathcal{S})-\operatorname{Lim}_{z \rightarrow x} f(z)$. if

The function $f: X \rightarrow Z$ is said to be $\mathcal{Y}$-very weakly $\mathcal{S}$-continuous in $x \in X$,

$$
f(x)=\left(\mathcal{S}_{\mathcal{Y}}^{t}\right)-\lim _{y \rightarrow x} f(y), \quad \text { for at least one } t \in T
$$

If

$$
f(x)=\left(\mathcal{S}_{\mathcal{Y}}^{\sqcap}\right)-\lim _{y \rightarrow x} f(y),
$$

we will say that $f$ is $\mathcal{Y}$-weakly $\mathcal{S}$-continuous at $x$, and finally, we will say that $f$ is $\mathcal{S}$-continuous at $x$, if

$$
f(x)=\mathcal{S}-\lim _{y \rightarrow x} f(y) .
$$

$D_{\mathcal{S}} f$ will denote the set of all $x$ 's for which $f(x)=\mathcal{S}$ - $\lim _{y \rightarrow x} f(y)$ fails. Any function which everywhere has all $\mathcal{S}_{\mathcal{Y}}^{t}$-limits (for $t \in T$ ) and is simultaneously $\mathcal{Y}$-very weakly $\mathcal{S}$-continuous for all $x$ 's, is called $(\mathcal{S}, \mathcal{Y})$-regular. By $D_{\mathcal{S}}^{\mathcal{Y}} f$ we shall denote the set of $\mathcal{Y}$-strong $\mathcal{S}$-discontinuity of $f$, i.e., the set of all $x \in X$, for which these among limits

$$
\left(\mathcal{S}_{\mathcal{Y}}^{t}\right)-\lim _{z \rightarrow x} f(z), \quad t \in T
$$

which exist, are pairwise different. 
Property 12. Assume that $\mathcal{S}$ is filtering, $(Z, \tau)$ is Hausdorff and that $f: X \rightarrow Z$. Then

1. $(\mathcal{S})-\lim _{y \rightarrow x} f(y)=g$ implies that $\left(\mathcal{S}_{\mathcal{Y}}^{t}\right)-\lim _{z \rightarrow x} f(y)=g$, for all $t \in T$,

2. $D_{\mathcal{S}}^{\mathcal{Y}} f \subset D_{\mathcal{S}} f$.

Proof. To prove 1 , note that the only nontrivial part is the uniqueness of $g$ as $\left(\mathcal{S}_{\mathcal{Y}}^{t}\right)$-limit of $f$ at $x$. Suppose contrary that for some $\bar{t} \in T$ and some $\bar{g} \neq$ $g:=(\mathcal{S})-\lim _{y \rightarrow x} f(y), f^{-1}\left(U_{\bar{g}}\right) \in S_{\mathcal{Y}}^{\bar{t}}(x)$, for each $\tau$-open $U_{\bar{g}}$ containing $\bar{g}$. By assumption, $f^{-1}\left(U_{g}\right) \in S_{\mathcal{Y}}^{\bar{t}}(x)$ for each open $U_{g} \ni g$. We can assume that $U_{g}$ and $U_{\bar{g}}$ are disjoint. Thus $f^{-1}\left(U_{g}\right) \cap f^{-1}\left(U_{\bar{g}}\right)=\varnothing$, which contradicts Property 8 in the light of the filtering assumption.

Assertion 2 is a straightforward consequence of 1.

TheOrem 4 (cf. [4], [14]). Assume that $(X, \rho)$ is a separable metric space and that for $f: X \rightarrow \mathbb{R}$, for a partition $\mathcal{Y}$ and for a local system $\mathcal{S}$ the following conditions are held:

1. $\mathcal{Y}=\left\{Y_{x}^{t}\right\}_{x \in X}^{t \in T}$ is regular,

2. $T$, the set of indices is finite,

3. $\mathcal{S}$ is $\mathcal{Y}$-retraceable,

4. $\mathcal{S}$ fulfills the intersection condition with a regularity guardian of $\mathcal{Y}$,

5. $f$ is $(\mathcal{S}, \mathcal{Y})$-regular.

Then the set $D_{\mathcal{S}}^{\mathcal{Y}} f$ is at most denumerable.

Proof. Let $T=\{1, \ldots, n\}$. Let us notice that in the case of an $(\mathcal{S}, \mathcal{Y})$-regular function, the set $D_{\mathcal{S}}^{\mathcal{Y}} f$ is reduced to the set on which the appropriate limits are pairwise different.

Let us focus on

$$
\begin{aligned}
A:=\{x \in X: & \left(\mathcal{S}_{\mathcal{Y}}^{1}\right) \lim _{z \rightarrow x} f(z)=f(x) \text { and } \\
& \left.\left(\mathcal{S}_{\mathcal{Y}}^{k}\right) \lim _{z \rightarrow x} f(z)<\left(\mathcal{S}_{\mathcal{Y}}^{k+1}\right)-\lim _{z \rightarrow x} f(z), \text { for } k \in\{1, \ldots, n-1\}\right\}
\end{aligned}
$$

and suppose that $A$ is not denumerable. The cases of other possible inequalities (or equivalently, nontrivial permutations of $T \cup\{f(x)\}$ ) could be treated by the same method. For $x \in A$ let $a(x):=\left\{a_{1}(x), \ldots, a_{k}(x), \ldots, a_{n-1}(x)\right\}$ be an $(n-1)$-tuple of rational numbers such that

$$
\left(\mathcal{S}_{\mathcal{Y}}^{k}\right) \lim _{z \rightarrow x} f(z)<a_{k}(x)<\left(\mathcal{S}_{\mathcal{Y}}^{k+1}\right)-\lim _{z \rightarrow x} f(z), \quad \text { for } \quad k \in\{1, \ldots, n-1\} .
$$




\section{GENERALIZED DISCONTINUITY OF REAL-VALUED FUNCTIONS}

By supposition, $A_{a}:=\{\xi \in A: a(\xi)=a\}$ would be uncountable for at least one $a=\left\{a_{1}, \ldots, a_{n-1}\right\} \in \mathbb{Q}^{n-1}$. Let

$$
\begin{aligned}
S_{\xi}^{a}:=\{\xi\} & \cup\left(Y_{\xi}^{1} \cap f^{-1}\left(-\infty ; a_{1}\right)\right) \\
& \cup \bigcup_{k=2}^{n-1}\left(Y_{\xi}^{k} \cap f^{-1}\left(a_{k-1} ; a_{k}\right)\right) \\
& \cup\left(Y_{\xi}^{n} \cap f^{-1}\left(a_{n-1} ;+\infty\right)\right) .
\end{aligned}
$$

It is easy to verify that $S_{\xi}^{a} \in S(\xi)$, if $\xi \in A_{a}$, in the light of assumption 3 .

By assumption 4, there is a regularity guardian $\mathcal{C}=\{C(x, y): x, y \in X\}$ of $\mathcal{Y}$ and a function $\delta: x \rightarrow(0, \infty)$ for which

$$
S_{x} \cap S_{y} \cap C(x, y) \neq \varnothing,
$$

if $\rho(x, y)<\min \{\delta(x), \delta(y)\}$. Without loss of generality, $\delta$ may be assumed to have only rational values. Thus, in the image of $A_{a}$, through the function $\delta$, there exists $\delta_{0} \in \mathbb{Q}$, such that $A_{a}\left(\delta_{0}\right):=\left\{z \in A_{a}: \delta(z)=\delta_{0}\right\}$ fails to be denumerable. Hence, one can choose $x, y \in A_{a}\left(\delta_{0}\right) \subset A_{a}$, so that $\rho(x, y)<\delta_{0}$, as $A_{a}$, being an uncountable subset of a second countable space, contains a condensation point of itself. But $S_{x}^{a} \cap S_{y}^{a} \cap C(x, y)=\varnothing$, by 1. This contradicts ([6), and thus 4 and the proof is complete.

If $\mathcal{S}$ is bilateral in $\mathbb{R}$, the limits referring to $\mathcal{S}^{-}$and $\mathcal{S}^{+}$are called unilateral $\mathcal{S}$-limits, the left and the right one, respectively. Thus, the following notation is legitimate.

$$
(\mathcal{S})-\lim _{y \rightarrow x+} f(y)=\left(\mathcal{S}^{+}\right)-\lim _{y \rightarrow x} f(y), \quad \mathcal{S}-\lim _{y \rightarrow x-} f(y)=\left(\mathcal{S}^{-}\right)-\lim _{y \rightarrow x} f(y) .
$$

The notion of $\mathcal{Y}_{\infty}$-very weak $\mathcal{S}$-continuity in the case of dimention $n=1$ is reduced to the notion of unilateral $\mathcal{S}$-continuity (left or right, respectively).

The forthcoming considerations refer to partition $\mathcal{Y}_{\square}$ defined in Example 9

Corollary 3. If, for $f: \mathbb{R} \rightarrow \mathbb{R}$ and $\mathcal{S} \in \mathbb{S}\left(\mathbb{R}, \mathcal{T}_{\text {nat }}\right)$, the assumptions of the above theorem hold, then $D_{\mathcal{S}} f=D_{\mathcal{S}}^{\mathcal{Y}_{\square}} f$ is at most denumerable.

Remark 3. For $f: \mathbb{R}^{n} \rightarrow \mathbb{R}, n>1$, the assumptions of the above theorem do not guarantee the denumerability of $D_{\mathcal{S}} f$.

EXAMPLE 15. Let us consider $\mathcal{S}_{\mathcal{I}}^{\circ}$, the local system in $\mathbb{R}^{2}$ in which $S \in S(x)$ only if $x \in S$ is an inner density point of all the intersections of $S$ with lines passing through $x$. Moreover, let $A \subset \mathbb{R}^{2}$ be an uncountable set having exactly two points incommon with every line in the plane [7]. It is not difficult to see that for $\mathcal{S}_{\mathcal{I}}^{\circ}$ and the function $\chi_{A}$, the conditions of Theorem 4 are fulfilled, while $D_{\mathcal{S}_{\mathcal{I}}^{\circ}} \chi_{A}=A$ is uncountable. 


\section{Applications}

Let $\lambda$ stand for Lebesgue measure in $\mathbb{R}^{n}, A$ be $\lambda$-measurable subset of $\mathbb{R}^{n}$ $(A \in \mathcal{L})$. We say that $x \in \mathbb{R}^{n}$ is a Lebesgue density point of $A$, in notation $x \in \Phi_{\mathcal{L}}(A)$ (cf. [1], 6], [12]), if

$$
\lim _{h \rightarrow 0} \frac{\lambda\left((A-x) \cap[-h ; h]^{n}\right)}{2^{n} h^{n}}=1 .
$$

Let $\omega^{\uparrow \omega}$ denote the collection of all increasing sequences of positive integers, ls - the upper limit of sequence of sets, and $\mathcal{N}$ - the ideal of Lebesgue null sets in $\mathbb{R}^{n}$.

Remark 4 ([1]). For $A \in \mathcal{L}$, the following conditions are equivalent.

1. $x \in \Phi_{\mathcal{L}}(A)$,

2. $\forall\left\{n_{k}\right\} \in \omega^{\uparrow \omega} \exists\left\{k_{p}\right\} \in \omega^{\uparrow \omega} \operatorname{ls}_{p \in \omega}\left([-1 ; 1]^{n} \backslash n_{k_{p}}(A-x)\right) \in \mathcal{N}$.

The proof uses Riesz theorem on stochastic convergence. This observation is due to Wilczyński, and in the one-dimensional case has led to the concept of $\mathcal{I}$-density point, here considered in $\mathbb{R}^{n}$ (cf. [1, [12, [14]). We say that $x \in \mathbb{R}^{n}$ is an $\mathcal{I}$-density point of $A \in \mathcal{B}$ (i.e., subset of $\mathbb{R}^{n}$ with Baire property), in notation $x \in \Phi_{\mathcal{I}}(A)$, provided that

$$
\underset{\left\{n_{k}\right\} \in \omega^{\uparrow \omega}}{\forall} \underset{\left\{k_{p}\right\} \in \omega^{\uparrow \omega}}{\exists} \operatorname{ls}_{p \in \omega}\left([-1 ; 1]^{n} \backslash n_{k_{p}}(A-x)\right) \in \mathcal{I},
$$

where (and hereafter) $\mathcal{I}$ is an ideal of meager subsets of $\mathbb{R}^{n}$.

Property 13 (cf. [12, [1]). Operators $\Phi_{\mathcal{L}}$ and $\Phi_{\mathcal{I}}$ mapping measurable subsets of $\mathbb{R}^{n}$ and sets with Baire property in $\mathbb{R}^{n}$ into their sets of Lebesgue density points and of $\mathcal{I}$-density points, respectively, are lower density operators on $(\mathcal{L}, \mathcal{N})$ and on $(\mathcal{B}, \mathcal{I})$, respectively.

Property 14. Let $S_{\mathcal{L}}(x):=\left\{S \in \mathbb{R}^{n}: x \in S\right.$ and $x \in \Phi_{\mathcal{L}}\left(S^{*}\right)$ for some $S^{*}$, $\mathcal{L}$-kernel of $S\}$ and $S_{\mathcal{I}}(x):=\left\{S \in \mathbb{R}^{n}: x \in S\right.$ and $x \in \Phi_{\mathcal{I}}\left(S^{*}\right)$ for some $S^{*}$, $\mathcal{B}$-kernel of $S\}$. The collections $\mathcal{S}_{\mathcal{L}}:=\left\{S_{\mathcal{L}}(x): x \in \mathbb{R}^{n}\right\}$ and $\mathcal{S}_{\mathcal{I}}:=\left\{S_{\mathcal{I}}(x)\right.$ : $\left.x \in \mathbb{R}^{n}\right\}$ are filtering local systems in $\left(\mathbb{R}^{n}, \mathcal{T}_{\text {nat }}\right)$.

Remark 5. System $\mathcal{S}_{\mathcal{L}} \sqcup \mathcal{S}_{\mathcal{I}}$ is not filtering in $\left(\mathbb{R}^{n}, \mathcal{T}_{\text {nat }}\right)$, which is a consequence of the existence of a co-meager Lebegue null set in $\mathbb{R}^{n}$.

TheOREM 5 (cf. [3, [14]). Both local systems $\mathcal{S}_{\mathcal{L}}$ and $\mathcal{S}_{\mathcal{I}}$ fulfill strong intersection condition with $\mathcal{C}_{\square}$ (Example 11) in the "max" metric on $\mathbb{R}^{n}$.

P r o of. For the case of measure, we will prove a little more than we should do in the light of $\operatorname{SIC}\left(\mathcal{C}_{\odot}\right)$, as for $\varepsilon>0$ we will define a function

$$
\delta^{\varepsilon}: \bigcup_{z \in \mathbb{R}^{n}}\left(\{z\} \times S_{\mathcal{L}}(z)\right) \rightarrow(0 ; \infty), \quad \delta^{\varepsilon}:\left(z, S_{z}\right) \mapsto \delta_{S_{z}}^{z, \varepsilon},
$$




\section{GENERALIZED DISCONTINUITY OF REAL-VALUED FUNCTIONS}

so that for all $x, y \in \mathbb{R}^{n}$ and $S_{x} \in S_{\mathcal{L}}(x), S_{y} \in S_{\mathcal{L}}(y)$, the intersection $S_{x} \cap S_{y} \cap$ $C_{\square}(x, y)$ is at least of measure $(1-\varepsilon) \lambda\left(C_{\square}(x, y)\right)$ if

$$
\|y-x\|<\min \left\{\delta_{S_{x}}^{x, \varepsilon}, \delta_{S_{y}}^{y, \varepsilon}\right\} .
$$

Let us note that considering only the case $y_{j} \geqslant x_{j}$, for all $j \leqslant n$, will not affect the generality of our method. Let us fix $\varepsilon>0, z \in \mathbb{R}^{n}, S_{z} \in S_{\mathcal{L}}(z)$ and $S_{z}^{*} \in \mathcal{L}$ such that $z \in S_{z}^{*} \subset S_{z}$ and $z \in \Phi_{\mathcal{L}}\left(S_{z}^{*}\right)$. By definition for $\bar{\varepsilon}>0$, there exists a positive $\eta\left(\bar{\varepsilon}, z, S_{z}^{*}\right)$, such that

$$
\lambda\left((z-\eta ; z+\eta)^{n} \cap S_{z}^{*}\right)>(2 \eta)^{n}(1-\bar{\varepsilon})
$$

if $0<\eta<\eta\left(\bar{\varepsilon}, z, S_{z}^{*}\right)$. Put

$$
\delta_{S_{z}}^{z, \varepsilon}:=\eta\left(\frac{\varepsilon}{2^{n+1}}, z, S_{z}^{*}\right)
$$

and assume that $\bar{\delta}:=\|y-x\|<\min \left\{\delta_{S_{x}}^{x, \varepsilon}, \delta_{S_{y}}^{y, \varepsilon}\right\}$. We have

$$
\bar{\delta}^{n} \leqslant \lambda\left(C_{\square}(x, y)\right) \leqslant 2^{n-1} \bar{\delta}^{n} .
$$

Let $B:=\times_{j=1}^{n}\left[y_{j}-\bar{\delta} ; y_{j}+\bar{\delta}\right]$. Obviously, $C_{\square}(x, y) \subset B$. By (주) we have

$$
\lambda\left(S_{y}^{*} \cap B\right) \geqslant\left(1-\frac{\varepsilon}{2^{n+1}}\right)(2 \bar{\delta})^{n} .
$$

Therefore,

$$
\begin{aligned}
\frac{\lambda\left(S_{y}^{*} \cap C_{\square}(x, y)\right)}{\lambda\left(C_{\square}(x, y)\right)} & \geqslant \frac{\lambda\left(S_{y}^{*} \cap B\right)-\left(\lambda(B)-\lambda\left(C_{\square}(x, y)\right)\right)}{\lambda\left(C_{\square}(x, y)\right)} \\
& \geqslant 1+\frac{\left(1-\frac{\varepsilon}{2^{n+1}}\right)(2 \bar{\delta})^{n}-(2 \bar{\delta})^{n}}{\lambda\left(C_{\square}(x, y)\right)} \\
& =1-\frac{\varepsilon \bar{\delta}^{n}}{2 \lambda\left(C_{\square}(x, y)\right)} \\
& \geqslant 1-\frac{\varepsilon}{2},
\end{aligned}
$$

the last inequality being a consequence of (10). By the similar argument we would prove that $\lambda\left(S_{x}^{*} \cap C_{\square}(x, y)\right)>\left(1-\frac{\varepsilon}{2}\right) \lambda\left(C_{\square}(x, y)\right)$.

Category part is an immediate consequence of [13, Theorem 3] and the Note on page 321 [13] applied to $\mathbb{R}^{n}$. Indeed, the characterization of an $\mathcal{I}$-dispersion point in $\mathbb{R}$ made by $\mathrm{E}$ a z arow ([11]) has an obvious $n$-dimensional analogue, cf. [14.

COROllary 4. Systems $\mathcal{S}_{\mathcal{I}}$ and $\mathcal{S}_{\mathcal{L}}$ in $\left(\mathbb{R}^{n}, \mathcal{T}_{\text {nat }}\right)$ are $\mathcal{Y}^{\square}$-decomposable and filtering. Thus, by Theorem 3 , they are $\mathcal{Y}^{\bullet}$-retraceable. 
COROLlaRY 5. Systems $\mathcal{S}_{\mathcal{I}}$ and $\mathcal{S}_{\mathcal{L}}$ in $\left(\mathbb{R}^{n}, \mathcal{T}_{\text {nat }}\right)$ satisfy the conditions of Theorem 4 (with $\mathcal{Y}^{\square}$ and $\mathcal{C}_{\square}$ ), hence the set of points of strong $\mathcal{S}_{\mathcal{L}}$-discontinuity of an $\mathcal{S}_{\mathcal{L}}$-regular function on $\mathbb{R}^{n}$, as well as the set of points of strong $\mathcal{S}_{\mathcal{I}}$-discontinuity of an $\mathcal{S}_{\mathcal{I}}$-regular function on $\mathbb{R}^{n}$ are at most denumerable.

CoRollary 6. Systems $\mathcal{S}_{\mathcal{I}}$ and $\mathcal{S}_{\mathcal{L}}$ considered in $\mathbb{R}$ fulfill the assumptions of Theorem 4 (with $\mathcal{Y}^{\square}$ and $\mathcal{C}_{\square}$ ), as well. Hence, by Corollary 3 , the sets $D_{\mathcal{S}_{\mathcal{I}}} f$ and $D_{\mathcal{S}_{\mathcal{L}}}$ f for the functions $\mathcal{S}_{\mathcal{I}}$-regular and $\mathcal{S}_{\mathcal{L}}$-regular, respectively, are at most denumerable.

\section{REFERENCES}

[1] CIESIELSKI, K.-LARSON, L.-OSTASZEWSKI, K.: I-density continuous functions, Mem. Amer. Math. Soc. 107 (1994), p. 133.

[2] ENE, V.: Real Functions-Current Topics, in: Lecture Notes in Math., Vol. 1603, SpringerVerlag, Berlin, 1995.

[3] FILIPCZAK, T.: Intersection conditions for some density and I-density local systems, Real Anal. Exchange 15 (1990), 170-192.

[4] GRANDE, M.: On the sums of unilaterally approximately continuous and approximately jump functions, Real Anal. Exchange 28 (2003), 623-630.

[5] GRÄTZER, G.: General Lattice Theory. Birkhäuser Verlag, Basel-Stuttgart, 1978.

[6] HOBSON, E. W.: The Theory of Functions of Real Variable and the Theory of Fourier's Series. Cambridge University Press, London, 1921.

[7] MAZURKIEWICZ, S.: O pewnej mnogości, która ma z każda prosta dwa i tylko dwa punkty wspólne, C. R. Varsovia 7 (1914), 382-384.

[8] SWIĄTKOWSKI, T.: O pewnym uogólnieniu pojęcia pochodnej, Zeszyty Nauk. Politech. Łódz. Mat. 149 (1972), 89-101.

[9] THOMSON, B.: Derivation bases on the real line II, Real Anal. Exchange 8 (1983), $278-442$.

[10] THOMSON, B.: Real Functions, in: Lecture Notes in Math., Vol. 1170, Springer-Verlag, 1980.

[11] WILCZYŃSKI, W.: A category analogue of the density topology, approximate continuity and the approximete derivative, Real Anal. Exchange 10 (1984-85), 241-265.

[12] WILCZYŃSKI, W.: Density topologies, in: Handbook of Measure Theory, Vol. I and II, North-Holland, Amsterdam, 2002, pp. 675-702.

[13] ZAJÍČEK, L.: Porosity, $\mathcal{J}$-density topology and abstract density topologies, Real Anal. Exchange 12 (1987), 313-326.

[14] ZDUŃCZYK, R.: Unilateral II-approximate limits of real functions, Real Anal. Exchange 34 (2008), 105-114.

Chair of Real Functions

Faculty of Mathematics and

Computer Science

Eódź University

22 Banacha st.

PL-90-238 Łódź

POLAND

E-mail: rafal@math.uni.lodz.pl 